\title{
Síndrome DRESS inducido por fármacos antituberculosos en un paciente con diabetes mellitus tipo 2
} Antituberculous drugs-induced DRESS syndrome in a patient with type 2 diabetes mellitus

\author{
Antonio Salas ${ }^{1,2}$, Víctor Mechán ${ }^{2,3}$, Félix Llanos ${ }^{1}$, Jorge Yoshihiro Nako ${ }^{4}$ \\ ${ }^{1}$ Médico Asistente de Neumología del Hospital Nacional Dos de Mayo, Lima, Perú. \\ ${ }^{2}$ Profesor de Medicina de la Universidad Naconal Mayor de San Marcos, Lima, Perú. \\ ${ }^{3}$ Médico Asistente de Hematologia del Hospital Nacional Dos de Mayo, Lima, Perú. \\ ${ }^{4}$ Estudiante de Medicina Humana de la Universidad Nacional Mayor de San Marcos, Lima, Perú.
}

\begin{abstract}
Resumen
Presentamos el caso de un paciente varón de 48 años de edad, con diagnóstico de diabetes mellitus tipo 2, no controlada, de diez años de evolución, a quien se le diagnosticó tuberculosis pulmonar mediante signos clínicos, radiográficos y cultivo en esputo positivo para Mycobacterium tuberculosis, sensible a drogas antituberculosas de primera línea. Recibió isoniacida, rifampicina, etambutol y pirazinamida. Dos meses después de iniciado el tratamiento presentó hipersensibilidad a medicamentos, con los siguientes signos y síntomas: rash dérmico generalizado, prurito generalizado, anemia Coombs positiva, eosinofilia y síntomas sistémicos, compatibles con el síndrome DRESS (drug rash with eosinophilia and systemic symptoms). Ante ello, se suspendió la medicación antituberculosa y se instaló tratamiento con antihistamínicos y corticoides sistémicos, con remisión y mejoría de síntomas. Posteriormente, recibió un esquema individualizado de tratamiento para tuberculosis consistente en medicamentos mínimamente hemato-hepatotóxicos, similar al indicado en pacientes inmunosuprimidos. Desde entonces presenta baciloscopias negativas.

Palabras clave: Diabetes mellitus, tuberculosis, Mycobacterium tuberculosis, hipersensibilidad a las drogas, antituberculosos, sindrome de eosinofilia-mialgia.
\end{abstract}

\section{Abstract}

A case of a 48 year-old male with uncontrolled type 2 diabetes mellitus for the past ten years who presented pulmonary tuberculosis by clinical, radiographic signs and Mycobacterium tuberculosis sputum culture, sensitive to first line treatment drugs, is reported. He received standard treatment with isoniazid, rifampicin, ethambutol, pyrazinamide showing two months later drug hypersensitivity consisting in generalized skin rash, pruritus, positive Coombs anemia, eosinophilia and systemic symptoms compatible with DRESS syndrome (drug rash with eosinophilia and systemic symptoms). The antituberculous drugs were suspended and systemic antihistaminic drugs and corticoids were administered with remission and improvement of symptoms. Afterwards individualized treatment scheme for tuberculosis consisting in minimal hemato-hepatotoxic drugs similar to those indicated to immunosuppressed patients was indicated. Baciloscopies have been negative since.

Key words: Diabetes mellitus, tuberculosis, Mycobacterium tuberculosis, drug hypersensitivity, antitubercular agents, eosinophiliamyalgia syndrome.

\section{An Fac med. 2012;73(2):159-64}

\section{INTRODUCCIÓN}

La prevalencia de tuberculosis (TBC) en pacientes adultos con diabetes mellitus tipo 2 (DM2) es de alrededor de $10 \%$, según diversos estudios ${ }^{(1-3)}$, mientras que la prevalencia de reacciones adversas a dichos medicamentos en pacientes con DM2, según estudios realizados en nuestro país, oscila entre
$5 \%$ y $10 \%{ }^{(4,5)}$. De otro lado, es conocida la susceptibilidad incrementada de pacientes con DM2 para adquirir TBC por mal control metabólico: hiperglicemias sostenidas, HbA1C (hemoglobina glicosilada) elevada, perfil lipídico con riesgo cardiovascular, tratamiento irregular y desarrollo de complicaciones crónicas ${ }^{(1,4)}$.
El síndrome DRESS, acrónimo de drug rash with eosinophilia and systemic symptoms, consiste en una reacción de hipersensibilidad a diferentes fármacos, entre ellos los medicamentos antituberculosos, produciendo rash, fiebre, eosinofilia, hepatitis y afectación sistémica ${ }^{(6,7)}$. Es un síndrome de consecuencias graves y potencialmente letales. Ante 
la aparición de los primeros síntomas del síndrome DRESS, es necesario suspender la medicación antituberculosa, lo que genera tratamientos irregulares o abandono terapéutico ${ }^{(1,4,5)}$.

Presentamos el caso de un paciente con DM2, con mal control metabólico, a quien se diagnosticó TBC y que luego de iniciada la terapia anti TBC presentó rash, eosinofilia y síntomas sistémicos compatibles con síndrome DRESS.

\section{CASO CLÍNICO}

Paciente varón de 48 años de edad con antecedente diagnóstico de DM2 desde hace diez años. Después del diagnóstico y durante los primeros cinco años estuvo en tratamiento con sulfonilureas 5 $\mathrm{mg} / \mathrm{d}$ ía, manteniendo concentraciones adecuadas de glicemia. En los siguientes años presentó niveles de glicemia elevados y disminución de peso; el tratamiento fue modificado a biguanidas $500 \mathrm{mg} /$ día y sulfonilureas $5 \mathrm{mg}$ dos veces al día, sin éxito, dado que persistían glicemias elevadas. Posteriormente, se hizo necesario el uso de insulina de acción rápida e intermedia por las hiperglicemias. Seis meses antes de su hospitalización, el paciente presentaba retinopatía diabética y parestesias distales en manos y pies; asimismo, sintomatología respiratoria compatible con TBC. La radiografía de tórax mostró dos cavitaciones en hemitórax izquierdo y se identificó bacilos ácido-alcohol resistentes en esputo y en cultivo de esputo Mycobacterium tuberculosis sensible a todos los medicamentos anituberculosos de primera línea. El paciente recibió tratamiento en un centro de salud del primer nivel de atención con el siguiente esquema: 2HRZE/4H2R2 [2 meses de medicación diaria con isoniacida $(\mathrm{H})$, rifampicina $(\mathrm{R})$, pirazinamida (Z), etambutoL (E) y 4 meses de medicación diaria con isoniacida $(\mathrm{H})$ y rifampicina (R)]. A los treinta días de iniciado el tratamiento presentó mejoría de la sintomatología de TBC y ganancia de peso.

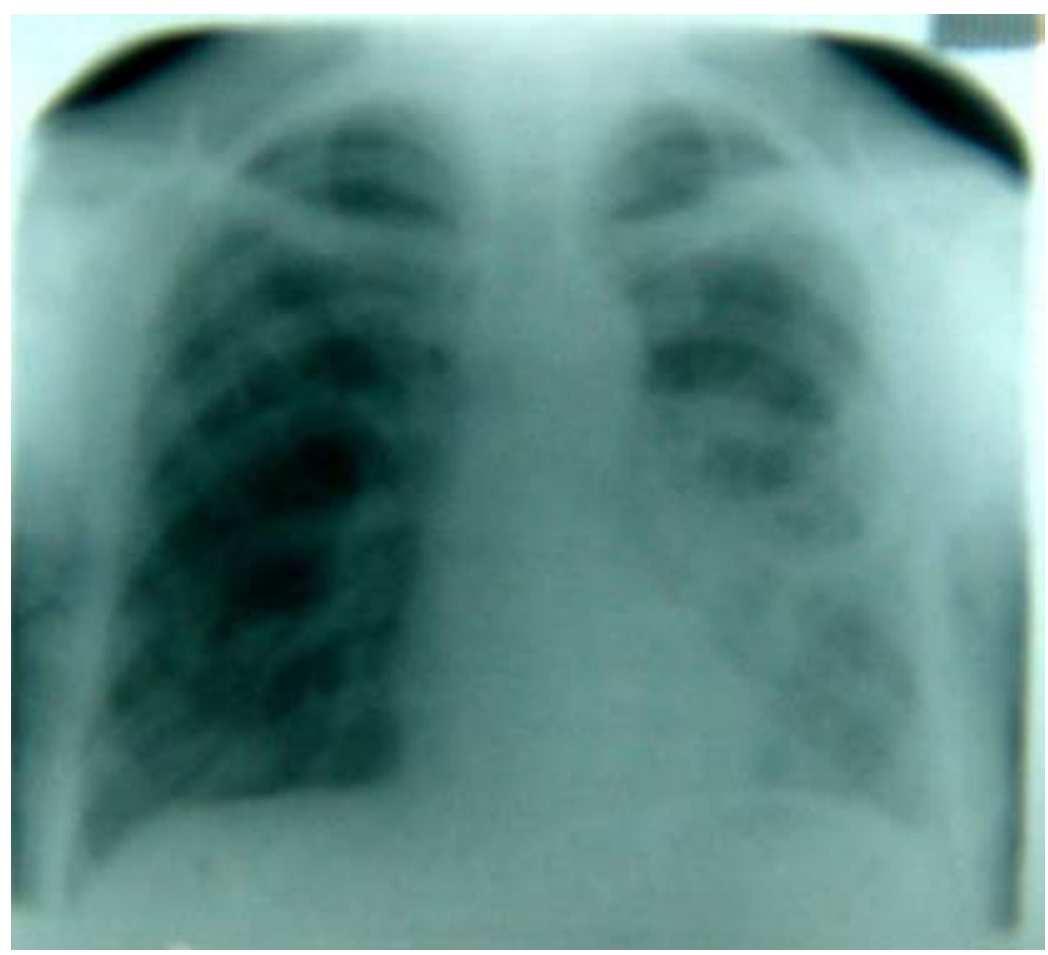

Figura 1. Fotoroentgen muestra en hemitórax izquierdo tractos fibrosos densos a predominio del hemitórax inferior; en el área parahiliar se evidencia patrón alveolar difuso; el seno costodiafragmático está obturado. En hemitórax derecho se observa escasos tractos fibrosos en base y área parahiliar.

Dos meses después de iniciado el tratamiento antituberculoso, el paciente mostró lesiones eritematosas generalizadas (exantemas) y prurito incoercible, por lo que, asumiéndose el desarrollo de una reacción adversa a los fármacos antituberculosos, se suspendió el tratamiento; el paciente recibió solo antihistamínicos y prednisona en los cuarenta y cinco días siguientes, con lo que el prurito y el eritema remitieron parcialmente. Por entonces, presentaba HbA1C 11,9\% y eosinofilia. Pocos días después el paciente presentó nuevamente sintomatología de TBC (fiebre y tos seca persistente), iniciándose un proceso de desensibilización a HRZE, manteniéndose la prednisona y los antihistamínicos, sin aparición de reacciones adversas.

Tras casi 2 meses sin tratamiento antituberculoso, el paciente fue hospitalizado a causa de dolor torácico pleurítico antero-izquierdo irradiado al dorso que se incrementaba con la inspiración, disnea, ortopnea, sudor nocturno, fiebre, hiporexia y heces verde-negruzcas cuatro veces al día. Se halló timpanismo, sin pasaje del murmullo vesicular en la base del hemitórax izquierdo, y posterior evidencia radiológica de hidroneumotórax izquierdo (sustracción pulmonar y nivel hidroaéreo). Ante esta sintomatología, se reinició el esquema antituberculoso estándar (2HRZE/4H2R2). El mismo día de iniciado el tratamiento, el paciente mostró prurito generalizado sin rash. Ante sospecha de infección pleural (fiebre, náuseas, vómitos, empleo de tubo de drenaje), se administró antibioticoterapia con ceftazidima y clindamicina, incrementándose la dosis de insulina $\mathrm{NPH}$.

Por persistencia de los síntomas (prurito generalizado), tres días después se suspendió el tratamiento antituberculoso, continuando con ceftazidima, clindamicina y antihistamínicos por vía parenteral. Un día después se agregaron 


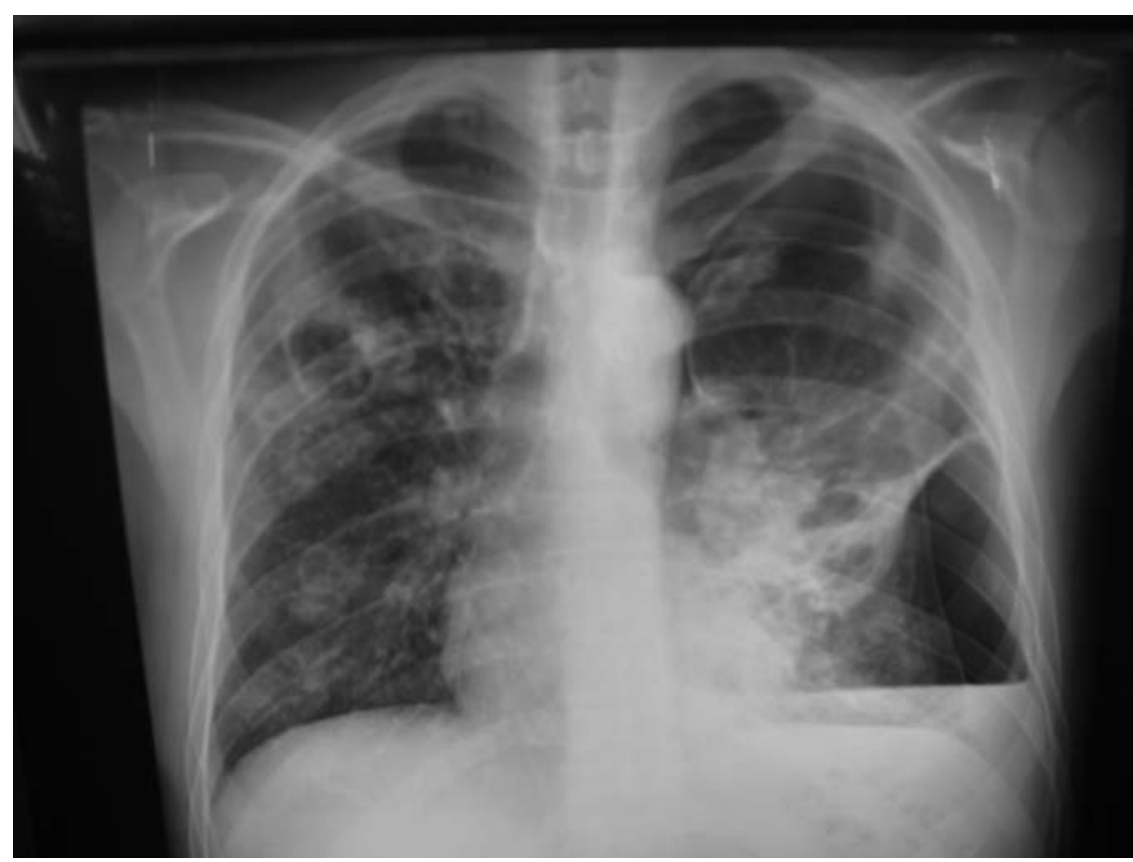

Figura 2. La radiografía muestra en hemitórax izquierdo tractos fibrosos y bulas enfisematosas en el lóbulo superior; en el área parahiliar, el patrón alveolar tiene bordes difusos; existe hidroneumotórax y nivel hidroaéreo en el tercio inferior, y derrame pleural. En hemitórax derecho existen imágenes reticulares subclaviculares, y cavitación de $2,5 \times 3 \mathrm{~cm}$ con paredes delgadas en el lóbulo superior.

al prurito, máculas torácicas e ictericia leve, descubriéndose lesiones cavitarias en hemitórax derecho (HTD), no vistas en radiografías previas. El paciente presentó ictericia leve (bilirrubinas totales $6 \mathrm{mg} \%$, a predominio indirecto $5,9 \mathrm{mg} \%)$, anemia hemolítica Coombs positiva, eosinofilia $(1267 / \mathrm{mL})$ y evidencia de hepatotoxicidad (TGO 74 U/L y TGP 55 U/L), diagnosticándose síndrome DRESS. Posteriormente, ante la suspensión de la medicación, los valores de bilirrubinas y transaminasas disminuyeron, aunque el prurito persistió en el área abdominal y lumbar.

Se inició un nuevo esquema antituberculoso, sustituyéndose INH y RFP (inductoras de anemias hemolíticas) por drogas mínimamente hemato-hepatotóxicas: etambutol, ciprofloxacino, cicloserina, amoxicilina/ácido clavulánico, ácido aminosalicílico y esteroides. El paciente mejoró paulatinamente, presentando controles negativos de TBC mediante baciloscopia, así como ganancia de peso.
Se desconoce la fisiopatología del síndrome DRESS, pero podrían intervenir diferentes mecanismos, tales como la predisposición genética, defectos de eliminación del fármaco empleado, alteraciones inmunológicas e infecciones asociadas como el herpes virus tipo 6 .

El mal control metabólico (hiperglicemias, retinopatía, neuropatía periférica, insuficiencia renal leve, $\mathrm{HbA1C}$ mayor de 7,5\%) y la cronicidad de la DM2 (existente en nuestro paciente) pueden explicar la rápida evolución clínico radiológica de TBC, similar a lo que ocurre en pacientes inmunodeprimidos. Las lesiones tuberculosas en pacientes con diabetes afectan los lóbulos pulmonares superiores e inferiores, a veces con fibrosis ${ }^{(8,9)}$. Algunos investigadores refieren daño por TBC en múltiples lóbulos, con escaso número de lesiones en lóbulos inferiores ${ }^{(10-12)}$. Restrepo y col. observaron que pacientes con coexistencia de TBC-DM2 presentaban tos, hemoptisis, fiebre y mayor número de cavitaciones pulmonares ${ }^{(13)}$, como se observó en nuestro paciente. Alisjahbana y col. encontraron que $22,2 \%$ de pacientes con TBC-DM2 tuvieron baciloscopias positivas hasta seis meses después del tratamiento ${ }^{(14)}$.

Atribuyendo a nuestro paciente mayor susceptibilidad para adquirir TBC por deficiencias en su sistema inmune, realizamos exámenes complementarios, cuyos resultados mostraron que no era resistente a drogas antituberculosas sino mas bien sensible a todos los medicamentos antituberculosos de primera línea. Dado que la literatura comunica que pacientes con DM2 con mal control metabólico pueden desarrollar una disminución de linfocitos T CD4 y $\mathrm{CD} 8$, asociados a mecanismos inmunorregulatorios deficientes ${ }^{(15,16)}$, comprobamos en este paciente que tales parámetros eran normales. Del mismo modo, la prueba azul de tetrazolio que valora la funcionalidad de polimorfonucleares fue normal.

Respecto a la asociación entre infecciones e hiperglicemias persistentes en pacientes con DM2, existen comunica- 
ciones que determinaron disminución de niveles de IL-6, IL-10 e IL-12 ${ }^{(4,17)}$ en pacientes con glicemias elevadas. Asimismo, lavados broncoalveolares de pacientes con coexistencia de TBCDM2 demostraron macrófagos alveolares con limitada actividad bactericida, condicionando estados de inmunosupresión ${ }^{(18)}$. Zhou, Tschopp y col. describen en pacientes con DM2 que ciertas infecciones liberan proteínas e interleuquinas inductoras de estados hiperglicémicos, promotoras de estados inflamatorios crónicos con resistencia a la insulina ${ }^{(19)}$.

Asimismo, las hiperglicemias y los productos finales de la glicosilación propician activación deficiente de los polimorfonucleares, existiendo correlación significativa entre los niveles elevados de $\mathrm{HbA1C}$ y una escasa actividad bactericida de estas células. Además, el sistema antioxidante involucrado en la actividad bactericida también se altera ${ }^{(17,19)}$. Los diabéticos con mal control metabólico desarrollan TBC con tasas elevadas de recaídas, fracasos y desarrollo de tuberculosis resistente a múltiples drogas, con alta sospecha de contagio intrahospitalario ${ }^{(4,5)}$.

El tipo de anemia más común en pacientes con TBC-DM2 es normocítica, normocrómica, asociada mayormente a granulomas en médula ósea, déficits nutricionales, malabsorción o mala utilización del hierro absorbido ${ }^{(20)}$. Aunque en pacientes con DM2-TBC las anemias hemolíticas autoinmunes son raras ${ }^{(21)}$, estas mayormente se asocian a TBC miliares ${ }^{(21,22)}$. Existe creciente evidencia de desarrollo de alteraciones autoinmunes (incluidas las anemias hemolíticas), inducidas por M. tuberculosis ${ }^{(23)}$. En algunos casos, las anemias hemolíticas autoinmunes son atribuidas a empleo de RFP ${ }^{(24,25)}$ o INH ${ }^{(26)}$, como parece haber sucedido en el presente caso, la cual cedió ante la interrupción del tratamiento anti tuberculoso. En las anemias hemolíticas producidas por fármacos anti tuberculosos, como la rifampicina, los mecanismos patogénicos no son conocidos con exactitud, aunque podrían ser causados por anticuerpos fijadores de complemento antirrifampicina. No obstante, la presencia de estos no siempre implica desarrollo de síntomas; contrariamente, pueden existir síntomas en ausencia de estos anticuerpos. La isoniacida raramente produce cuadros de hemólisis ${ }^{(26)}$. La anemia hemolítica Coombs positiva de grado leve encontrada en nuestro paciente forma parte del síndrome DRESS ${ }^{(6,7)}$. La definición de reacción adversa a medicamentos incluye reacciones relacionadas con hepatotoxicidad o hepatitis ${ }^{(27)}$.

Los síntomas sistémicos de nuestro paciente, así como las alteraciones funcionales dermatológicas y hepáticas, se resolvieron empleando medicación antituberculosa mínimamente hematohepatotóxica, similar a la empleada en inmunosuprimidos severos ${ }^{(28,29)}$.

Entre los factores de riesgo del síndrome DRESS se encuentran la edad, alcoholismo, medicación hepatotóxica concomitante, empleo de drogas intravenosas, intolerancia previa a isoniacida, embarazo y puerperio temprano, entre otros ${ }^{(4,5)}$. Los siguientes factores de riesgo incrementan la susceptibilidad para adquirir $\mathrm{TBC}$ en pacientes con DM2: hiperglicemias sostenidas, desnutrición, mayores de 40 años, complicaciones crónicas de DM2, suspensión del tratamiento antituberculoso, niveles elevados de HbA1C, terapéutica inadecuada, alcoholismo o presencia de hepatopatías ${ }^{(27)}$. Para casos como el publicado, el rápido reemplazo de esquemas antituberculosos estándar por otros semejantes a los empleados en inmunosuprimidos es opción a considerar, a fin de impedir la veloz diseminación de la TBC inducida por terapéutica suspendida a causa de hipersensibilidad medicamentosa ${ }^{(30)}$.

Las manifestaciones clínicas del síndrome DRESS suelen aparecer entre las 2 y 8 semanas de exposición al fármaco ${ }^{(31,32)}$, como ocurrió en nuestro paciente, que presentó los síntomas a las 8 semanas de tratamiento antituberculoso. Las lesiones dérmicas en el síndrome DRESS pueden ir desde un exantema maculopapular confluente y pruriginoso céfalo caudal, descamativo, hasta la confluencia de lesiones y eritrodermia; dichas lesiones pueden evolucionar a dermatitis exfoliativa. El edema facial periorbitario es frecuente y junto con la fiebre son signos iniciales de este síndrome ${ }^{(33)}$. El inicio de la presentación de síntomas en nuestro caso fue con exantema generalizado.

Entre las manifestaciones no dermatológicas del síndrome DRESS destacan: fiebre, adenopatías, alteraciones hematológicas (eosinofilia, leucocitosis, anemia hemolítica) y daño visceral (hepatitis, nefritis). Nuestro paciente presentó de manera progresiva las manifestaciones descritas, aunque la función renal fue conservada en todo momento y además no se evidenció adenopatías. Similares hallazgos fueron comunicados en un paciente de un hospital especializado de Lima-Perú, también con diagnóstico de TBC y síndrome DRESS, en quien destacaba una marcada eosinofilia y elevación de transaminasas ${ }^{(34)}$. Se ha descrito efectos tóxicos en el endotelio vascular, con alteraciones cardiacas, gastrointestinales, pulmonares y renales en eosinofilias elevadas ${ }^{(35)}$.

Se desconoce la etiología del síndrome DRESS. Se ha planteado la intervención de diferentes mecanismos, tales como, predisposición genética, defectos de eliminación del fármaco empleado, desnutrición, deficiencias o alteraciones enzimáticas, alteraciones inmunológicas e infecciones asociadas, como el herpes virus tipo $6^{(31)}$. De los factores mencionados, nuestro paciente ingería drogas antituberculosas y fue considerado en todo momento como inmunosuprimido, dada su condición de paciente diabético con mal control metabólico. Entre los fármacos descritos que inducen síndrome DRESS se encuentran: abacavir, dapsona, nevirapina, alopurinol, diltiazem, atenolol, sales de oro, fenobarbital, azatioprina, isoniacida, fenitoína, captopril, lamotrigina, sulfasalazina, carbamazepina, sulfonamidas, clomipramina, minociclina, trimetropima ${ }^{(36)}$. 
Acerca de la fisiopatología del síndrome DRESS, se plantea una reacción de hipersensibilidad alérgica en la que los medicamentos actúan directamente como antígenos o indirectamente como haptenos, desencadenando producción de anticuerpos. Se ha planteado la ausencia de la enzima epóxido hidrolasa (necesaria para detoxificar los metabolitos de las drogas que desencadenan respuesta inmune celular) ${ }^{(34,35)}$. El diagnóstico diferencial del síndrome DRESS incluye el síndrome Steven Johnson, enfermedad de Kawasaki, enfermedad de Still, infecciones virales (Epstein Bar, VIH, citomegalovirus, hepatitis), sepsis, linfomas, leucemias.

Respecto al tratamiento del síndrome DRESS, la primera medida es suspender el medicamento sospechoso de estar produciendo hipersensibilidad. Los corticoides sistémicos representan el tratamiento más aceptado, indicado sobre todo cuando hay compromiso sistémico. La recuperación por lo general es completa desde la suspensión del fármaco desencadenante y ante el tratamiento instaurado; existe mayor mortalidad en quienes presentan fallo hepático, insuficiencia renal y sepsis. En el presente caso, el paciente continuó su tratamiento antituberculoso con esquema individualizado, dado el antecedente de hipersensibilidad.

El síndrome DRESS representa una reacción grave y potencialmente letal de hipersensibilidad a diferentes fármacos, entre ellos los fármacos antituberculosos, como en el presente caso. Se debe sospechar este síndrome ante la presencia de rash, fiebre, eosinofilia, hepatitis o afectación sistémica.

\section{AGRADECIMIENTOS}

\author{
Dr. Juan Sosa Páucar, Jefe del Servicio \\ de Neumología del HN2Mayo de Lima, \\ y al Dr. José Ramos Castillo, por su co- \\ laboración en el perfeccionamiento del \\ escrito.
}

\section{REFERENCIAS BIBLIOGRÁFICAS}

1. Ezung T, Devi NT, Singh NT, Singh TB. Pulmonary tuberculosis and diabetes mellitus - a study. J Indian Med Assoc. 2002;100 (6):376, 378-9.

2. Kim SJ, Hong YP, Lew WJ, Yang SC, Lee EG. Incidence of pulmonary tuberculosis among diabetics. Tuber Lung Dis. 1995;76 (6):529-33.

3. Delgado JL, Seclén SN, Gotuzzo E. Tuberculosis en pacientes con diabetes mellitus: un estudio epidemiológico y clínico en el Hospital Nacional Cayetano Heredia. Rev Med Hered. 2006;17 (3):132-40.

4. Bonilla C, Suárez PG, Portocarrero J, Gutarra K. Patrón de presentación de reacciones adversas a fármacos antituberculosos en tratamientos directamente observados, Perú 1991-2000. En: Dirección General de Salud de las Personas / Programa Nacional de Control de Enfermedades Transmisibles / Programa Nacional de Control de la Tuberculosis - Ministerio de Salud del Perú. Tuberculosis en el Perú: Informe 2000. Lima: Tarea Asociación Gráfica Educativa; 2001:107-20.

5. Rodríguez DC, Ramírez JH, Pacheco VH, Salazar LE, Casalino E, Lizarzaburu L. Kemper R. Efectos adversos de consecuencia fatal por tratamiento con fármacos antituberculosos. Rev Med Hered. 2002;13 (4):148-52.

6. Queyrel V, Catteau B, Michon-Pasturel U, Fauchais AL, Delcey V, Launay D, Legout L, Hachulla E, Hatron PY, Devulder B. DRESS (Drug Rash with Eosinophilia and Systemic Symptoms) syndrome after sulfasalazine and carmazepine: report of two cases. Rev Med Interne. 2001;22 (6):582-6.

7. Lee JH, Park HK, Heo J, Kim TO, Kim GH, Kang $\mathrm{DH}$, Song GA, Cho M, Kim DS, Kim HW, Lee CH. Drug Rash with Eosinophilia and Systemic Symptoms (DRESS syndrome) induced by celecoxib and anti-tuberculosis drugs. J Korean Med Sci. 2008;23 (3):521-5.

8. Pérez-Guzmán C, Torres-Cruz A, Villarreal-Velarde $\mathrm{H}$, Salazar-Lezama MA, Vargas MH. Atypical radiological images of pulmonary tuberculosis in 192 diabetic patients: a comparative study. Int J Tuberc Lung Dis. 2001;5 (5):455-61.

9. El-Warraki S. The pattern and behavior of pulmonary tuberculosis in diabetic patients. Dis Chest. 1963; 43:582-6.

10. González-Hernández Y, Sada Diaz E, EscobarGutiérrez A, Muños Torrico M, Torres Rojas M. Asociación de tuberculosis y diabetes mellitus: Mecanismos inmunológicos involucrados en la susceptibilidad. Rev Inst Nac Enf Resp. 2009;22 (1):48-55.

11. Morris JT, Seaworth BJ, McAllister CK. Pulmonary tuberculosis in diabetics. Chest. 1992;102 (2):539-41.

12. Perez-Guzman C, Torres-Cruz A, Villarreal-Velarde $\mathrm{H}$, Vargas $\mathrm{MH}$. Progressive age-related changes in pulmonary tuberculosis images and the effect of diabetes. Am J Respir Crit Care Med. 2000;162 (5):1738-40.

13. Restrepo BI, Fisher-Hoch SP, Crespo JG, Whitney E Perez A, Smith B, McCormick JB; Nuevo Santander Tuberculosis Trackers. Type 2 diabetes and tuberculosis in a dynamic bi-national border population. Epidemiol Infect. 2007;135 (3):483-91.

14. Alisjahbana B, Sahiratmadja E, Nelwan EJ, Purwa AM, Ahmad Y, Ottenhof IH, Nelwan RH, Parwatti I, vandermer JW, van Crevel R. The effect of type 2 diabetes mellitus on the presentation and treatment response of pulmonary tuberculosis. Clin Infect Dis. 2007;45:428-35.
15. Musa BOP, Onyemelukwe GC, Hambolu JO, Bakari AG, Anumah FE. Cell-mediated immunity in type 2 diabetes mellitus patients in diabetic ketoacidosis, patients with controlled type 2 diabetes mellitus and healthy control subjects. J Med Medic Sci. 2010:1 (7); 290-5.

16. Joshi N, Caputo GM, Weitekamp MR, Karchmer AW. Infections in patients with diabetes mellitus. NEJM. 1999;341(25):1906-12.

17. Chen YH, Lin SJ, Lin FY, Wu TC, Tsao CR, Huang $\mathrm{PH}$, Liu PL, Chen YL, Chen JW. High glucose impairs early and late endothelial progenitor cells by modifying nitric oxide-related but not oxidative stress-mediated mechanisms. Diabetes. 2007;56 (6):1559-68.

18. Wang CH, Yu CT, Lin HC, Liu CY, Kuo HP. Hypodense alveolar macrophages in patients with diabetes mellitus and active pulmonary tuberculosis. Tuber Lung Dis. 1999;79 (4):235-42.

19. Zhou R, Tardivel A, Thorens B, Choi I, Tschopp J. Thioredoxin-interacting protein links oxidative stress to inflammasome activation. Nature Immunol. 2010;11(2):136-40.

20. Singh DK, Winocour P, Farrington K. Erythropoietic stress and anemia in diabetes mellitus. Nat Rev Endocrinol. 2009;5 (4):204-10.

21. Kuo PH, Yang PC, Kuo SS, Luh KT. Severe immune hemolytic anemia in disseminated tuberculosis with response to antituberculosis therapy. Chest. 2001;119 (6):1961-3.

22. Turgut M, Uzun O, Kelkitli E, Özer O. Pulmonary tuberculosis associated with autoimmune hemolytic anemia: an unusual presentation. Turk J Hematol. 2002;19 (4):477-80.

23. Riebeling-Navarro C, Madrid-Marina V, CamarenaMedellin B, Peralta-Zaragoza O, Barrera R. Agentes infecciosos y enfermedades autoinmunes. Salud Pública Méx. 1992;34 (3):342-51.

24. Curry FJ. National Tuberculosis Center and California Department of Health Service. Tuberculosis Drug Information Guide. Second Edition. 2008; pp: 155.

25. Ahrens N, Genth R, Salama A. Belated diagnosis in three patients with rifampicin-induced immune haemolytic anaemia. Brit J of Haematol. 2002;117 (2):441-3.

26. Robinson MG, Foadi M. Hemolytic anemia with positive Coombs' test. Association with isoniazid therapy. JAMA. 1969;208 (4):656-8.

27. Gholami K, Kamali E, Hajiabdolbaghi M, Shalviri G. Evaluation of anti-tuberculosis induced adverse reactions in hospitalized patients. Pharm Pract. 2006;4 (3):134-8

28. Wada M. Effectiveness and problems of PZAcontaining 6-month regimen for the treatment of new pulmonary tuberculosis patients. Kekkaku. 2001;76 (1):33-43.

29. Kameda K, Kawabata S, Masuda N. Follow-up study of short course chemotherapy of pulmonary tuberculosis complicated with diabetes mellitus. Kekkaku. 1990;65 (12):791-803.

30. Amado Tineo J. Factores de riesgo asociados a multi-drogorresistencia en pacientes con tuberculosis sin infección por VIH internados en el Hospital Nacional Dos de Mayo, Lima-Perú [tesis]. Facultad de Medicina. Universidad Nacional Mayor de San Marcos, 2007.

31. Castagnino J, Musella R, Palmero D. Sindrome DRESS inducido por fármacos anti tuberculosis. Rev Am Med Resp. 2011;3:141-6.

32. Tas S, Simonart T. Management of Drug Rash with Eosinophilia and Systemic Symptoms (DRESS Syndrome): An Update. Dermatol. 2003;206:353-6. 
33. Walsh SA, Creamer D. Drug reaction with eosinophilia and systemic symptoms (DRESS): a clinical update and review of current thinking. Clin Exp Dermatol. 2011;36: 6-11.

34. Elguera-Falcón F, Juárez-García M. Sindrome DRESS durante tratamiento antituberculoso. Rev Soc Peru Med Int. 2011;24:207-11.
35. Cervigón I, Sandin S, Pérez C, Bahilo C, Vélez C, Garcia D. Sindrome de DRESS (Drug Rash with Eosinophilia and Systemic Symptoms) por sulfonamidas. Med Cutan Iber Lat Am. 2006;34 (3):120-6.

36. Wolkenstein P, Revuz J. Drug-induced severe skin reactions - incidence, management and prevention. Drug Safety. 1995;13:56-68.
Artículo recibido el 21 de junio de 2011 y aceptado para publicación el 8 de mayo de 2012.

\section{Correspondencia:}

Antonio Salas e-mail: jaslk3025@yahoo.com

Victor Mechán e-mail: victor.mechan@gmail.com 